\title{
Cross Layer QoS Aware Scheduling based on Loss-Based Proportional Fairness with Multihop CRN
}

\author{
K. Saravanan ${ }^{1, *}$, G. M. Tamilselvan ${ }^{2}$ and A. Rajendran ${ }^{3}$ \\ ${ }^{1}$ Department of ECE, KPR Institute of Engineering and Technology, Coimbatore, India \\ ${ }^{2}$ Department of IT, Sri Krishna College of Technology, Coimbatore, India \\ ${ }^{3}$ Department of ECE, Karpagam College of Engineering, Coimbatore, India \\ *Corresponding Author: K. Saravanan. Email: sarakrish82@gmail.com \\ Received: 08 June 2021; Accepted: 22 July 2021
}

\begin{abstract}
As huge users are involved, there is a difficulty in spectrum allocation and scheduling in Cognitive Radio Networks (CRNs). Collision increases when there is no allocation of spectrum and these results in huge drop rate and network performance degradation. To solve these problems and allocate appropriate spectrum, a novel method is introduced termed as Quality of Service (QoS) Improvement Proper Scheduling (QIPS). The major contribution of the work is to design a new cross layer QoS Aware Scheduling based on Loss-based Proportional Fairness with Multihop (QoSAS-LBPFM). In Medium Access Control (MAC) multi-channel network environment mobile nodes practice concurrent broadcast between several channels. Acquiring the advantage of introduced cross layer design, the real-time channel conditions offered by Cognitive Radio (CR) function allows adaptive sub channel choice for every broadcast. To optimize the resources of network, the LBPFM adaptively plans the radio resources for allocating to diverse services without lessening the quality of service. Results obtained from simulation proved that QoSAS-LBPFM provides enhanced QoS guaranteed performance against other existing QIPS algorithm.
\end{abstract}

Keywords: Network efficiency; distributed architecture; mobile ad hoc networks; Cognitive Radio Networks (CRNs); QoS improvement; scheduling

\section{Introduction}

In Cognitive Radio Network (CRN), minor users are permitted to utilize unused spectrum that is allocated to the licensed or major users [1]. Cognitive radio is the main theory behind CRN [2]. The CR has the ability to analyze the environment and implement the broadcast. Every secondary user utilizes the unused or under-used spectrum without disrupting the operation of primary users [3]. The main idea of opportunistic scheduling standard in cognitive wireless networks is to utilize the time varying spectrum to enhance the throughput and reduce the hindrance to major users. Most of the present opportunistic scheduling methods in CRNs concentrates on improvising the throughput [4,5] and affording justice between various users [6]. While varied types of CRNs exists, minor users with varied network qualities also exists in the similar network. In future, all the devices will be CR enabled due to the advancement in

This work is licensed under a Creative Commons Attribution 4.0 International License, which permits unrestricted use, distribution, and reproduction in any medium, provided the original work is properly cited. 
the field of hardware. Ad hoc networks are known as famous network formation in the concept of IoT after their autonomy from pre-defined network infrastructure. Provided that nodes in the ad hoc network are capable of self-organized conversation, an adequate device-to-device transmission network can be deployed automatically wherever required. Current research indicated that cognitive radio has considerable impacts on higher layer operations in Wireless Node (WN), particularly Mobile Adhoc Network (MANET). MANET helps wireless devices in establishing dynamic network without utilizing stable structure. Hence, cognitive radio method plays a vital role in serving nodes to create networks based on their capability of vibrant access. Multi-channel Medium Access Control (MAC) protocol permit varied users to transmit via diverse pathways concurrently without interrupting others [7]. The delay and throughput is enhanced in simultaneous transmission mode by enabling varied users to transmit the data. In Zhang et al. [8-10] the authors have introduced a Cognitive Radio Enabled Multi-Channel MAC protocol that depends on unique control channel broadcasting mode. Nevertheless, most of the research concentrates on sensing the channel, selecting and providing service through a simple model that mainly ignores QoS scheduling such as delay, packet delivery ratio, overhead ratio, collision probability, throughput, of diverse applications. The main issues of real time communication systems are reliability, safety and time limit guaranteed data transmission. This motivates to design a new scheduling algorithm for cross layer in CRN.

An proficient pathway scheduling method is required to exploit spectrum holes and minimize the disturbance to major users. In order to accomplish effective usage of resources of the network, there is a need for an effectual packet scheduling approach that choose packet to forward or to put in queue. Main requirement for cognitive radio networks is to enhance the utilization of existing spectrum and plan for lot more packets. Moreover, in distributed CRNs, several scheduling issues arises because of the decentralized framework. In order to solve these problems, the work proposed a new cross QoS Aware Scheduling based on Loss-based Proportional Fairness with Multihop (QoSAS-LBPFM). Using prior information of usage of frequency and the condition of radio link of the physical layer, the MAC layer provides appropriate multi-channel choice for information promoting and broadcasting. Moreover, the LBPFM method consider the CR MANET's multi-hop nature along with the information about real time QoS while planning radio resources for diverse services to have better utilization of overall network. The manuscript is prepared as: Section II explains the correlated research methods on scheduling in CRNs. In Section III, the introduced QoSAS-LBPFM in CR MANET is illustrated. Section IV includes the investigational results. In Section V, the work is concluded with future enhancement.

\section{Related Work}

This part explains the outline of CR networks and its recent advances in resource allocation techniques. In Liang et al. [11], described a methodical outline on CR networking and communications considering the major tasks of the MAC, PHY and network layers occupied in a CR design and their relations across the layers. Though building purely cognitive radios and wireless networks is a easy job it needs combined contribution of different research group of people, together with theory of communications, engineering in networking, signal processing, game theory, combined design of software-hardware and design of reconfigurable antenna and radio-frequency.

In Wang et al. [12], considered prerequisite of QoS in an ad hoc CRN with underlay of spectrum. Here the streaming and information traffics are accounted. Streaming traffic needs reduced probability of session outage whereas non-real-time data traffic requires reduced average throughput. For this, a system of packet scheduling with joint connection admission is introduced. In Luan et al. [13], introduced scheduling of resources in relay-assisted CRN with the Orthogonal Frequency-Division Multiple Access (OFDMA) system that is implemented to deal more than user configuration. Here, the authors have discussed about 
feasibility above assignment of relay, distribution of subcarrier, individual power control, and heterogeneous QoS provisioning. In Sun et al. [14], considered the Cooperative Sensing Scheduling QoS guarantee (CSS-Q issue) for developing a CRN with effective energy. Here the intrinsic formation of the CSS-Q issue is investigated to analyze several functional properties. Depending on these properties, an effective method is anticipated to resolve the problem. The main problem in sensing of mutual spectrum is to identify how to allocate SUs (Secondary Users/minor users) for sensing diverse crucial pathways so that SUs can acquire superior stability among accuracy of sensing and examination of probable "spectrum holes". It is very difficult when the major channels need heterogeneous detection probabilities for the protection of incumbent. The QoS transmission becomes difficult for smart grid applications that are based on Wireless Sensor Network (WSN) due to interference of electromagnet, noise of equipment, effects in multi-path and smart grid surroundings' obstructions. To solve these problems, authors have introduced a cross-layer structure that depends on cognitive communication in Shah et al. [15]. The introduced model utilizes the promising technology of CR to diminish the spectrum bands that are prone to noise and congestion, to provide a trustworthy with huge capacity links for smart grids wireless communication.

In Homayounzadeh et al. [16] the difficulty of provisioning of QoS for the Real-Time secondary traffic (RTtraffic) in CRNs is examined. Sustaining the RT traffic in cognitive radio network is a difficult work that needs complex requirements in diverse network characteristic. A new method of scheduling packet is anticipated that effectively provides the requirements of QoS for RT Secondary Users (SUs). The proposed framework also considers the deviations in the wireless frequency channels of Signal to Noise Ratio (SNR) that result in channels' variable transmission rate. In Tumuluru et al. [17], introduced an opportunistic spectrum-scheduling method for multi-channel CRNs. Here, the activity of primary user along with the quality of channel (SNR at the receiving secondary user) varies for every period.

In Yu et al. [18], examined the QoS differential planning issue in the smart grid communication networks that are based on CR networks. The scheduler is accountable for the management of resources of spectrum and organizing the transmission $f$ information among the Smart Grid Users (SGUs). For guaranteed differential QoS, the SGUs are allocated with diverse priorities depending on their functions and present situation of smart grid.

In Ali et al. [19], the licensed channel availability based on the activities of primary/major users like patterns of traffic are examined and proposed a priority-based secondary user call admission and method for allotment of channel depending on priority-based vibrant channel reservation system. Nevertheless, the CR- Internet of Things (IoT) cannot accommodate entire users because of less vibrant availability of resource. In Zhang et al. [20] implemented a deep Q -learning technique to construct feasible information broadcasting scheduling method for cognitive vehicular networks to reduce the cost of broadcasting and completely using different resources and modes of communication. Nevertheless, extremely vibrant topology with time-changing spectrum states in vehicular networks that are based on CR introduced some issues that is to be solved.

Spectrum allocation with scheduling is complicated in cognitive radio networks because of greater number of users. Collision is increased in cognitive radio network when there is no spectrum allocation which results in huge dropping rate and decreased performance in network. In order to address the problems with appropriate spectrum allocation a novel method is proposed in Rathika et al. [21], termed as QIPS (QoS improvement proper scheduling). The method is examined for mobile nodes in handoffs in the CRN. In this framework, the base station is extended to handle dynamic mobile nodes, nodes that are connected and disconnected, examining the performance of stable and mobile nodes in CRN with the help of QoS parameters. Tab. 1 shows the comparative analysis of existing methods. 
Table 1: Comparative analysis of existing methods

\begin{tabular}{|c|c|c|c|}
\hline Authors & Approaches & Advantages & Disadvantages \\
\hline $\begin{array}{l}\text { Bin Wang } \\
\text { et al. } \\
(2011) \\
{[12]}\end{array}$ & $\begin{array}{l}\text { joint connection } \\
\text { admission and packet } \\
\text { scheduling method is } \\
\text { introduced }\end{array}$ & $\begin{array}{l}\text { Attains reduced and reasonable } \\
\text { session outage probability for } \\
\text { streaming traffic, increased } \\
\text { throughput with reasonable } \\
\text { transmission delay for data } \\
\text { traffic, the achieved } \\
\text { performance is nearer to the } \\
\text { feasible scheduling }\end{array}$ & $\begin{array}{l}\text { High Computational } \\
\text { Complexity }\end{array}$ \\
\hline $\begin{array}{l}\text { Xiangxia } \\
\text { Sun et al. } \\
(2011) \\
{[14]}\end{array}$ & $\begin{array}{l}\text { CSS-Qissue is analyzed } \\
\text { for constructing CRNs } \\
\text { that are energy-efficient }\end{array}$ & $\begin{array}{l}\text { Achieves higher detection } \\
\text { probability }\end{array}$ & $\begin{array}{l}\text { Disadvantages are the need for a } \\
\text { prior knowledge of PU signal } \\
\text { characteristics and high } \\
\text { computational complexity. }\end{array}$ \\
\hline $\begin{array}{l}\text { Ghalib A. } \\
\text { Shah et al. } \\
\text { (2013) } \\
{[15]}\end{array}$ & $\begin{array}{l}\text { A sub-optimal distributed } \\
\text { control method (DCA) is } \\
\text { introduced for effective } \\
\text { QoS support }\end{array}$ & $\begin{array}{l}\text { Outcome of experiments } \\
\text { indicated that when there is a } \\
\text { increase in lower priority class } \\
\text { flow, it does not change the } \\
\text { high priority class performance } \\
\text { of any attribute }\end{array}$ & $\begin{array}{l}\text { High Computational } \\
\text { Complexity }\end{array}$ \\
\hline $\begin{array}{l}\text { Tumuluru } \\
\text { et al. } \\
\text { (2011) } \\
{[17]}\end{array}$ & $\begin{array}{l}\text { new Spectrum-Scheduling } \\
\text { Scheme }\end{array}$ & $\begin{array}{l}\text { The introduced scheduling } \\
\text { method provides less overhead } \\
\text { on with increased throughput }\end{array}$ & $\begin{array}{l}\text { The overhead of scheduling } \\
\text { may have high impact on the } \\
\text { performance of the system }\end{array}$ \\
\hline $\begin{array}{l}\text { Rong Yu } \\
\text { et al. } \\
(2016) \\
{[18]}\end{array}$ & $\begin{array}{l}\text { An Adaptive Dynamic } \\
\text { Programming Approach } \\
\text { for QoS Differential } \\
\text { Scheduling in } \\
\text { Cognitive-Radio-Based } \\
\text { Smart Grid Networks }\end{array}$ & $\begin{array}{l}\text { It Achieves better results in } \\
\text { QoS parameters }\end{array}$ & $\begin{array}{l}\text { The major disadvantage is that } \\
\text { the value of output is stored } \\
\text { number of times without getting } \\
\text { utilized in subsequent execution } \\
\text { of sub problem that results in } \\
\text { high utilization of memory. }\end{array}$ \\
\hline
\end{tabular}

\section{Proposed Methodology}

A novel cross layer QoS aware scheduling method and Loss based Proportional Fairness with Multi-Hop (LBPFMH) is proposed in this work for CRMANET. For data forwarding and transmission, suitable multichannel selections provided effectively by MAC layer, with a radio link and frequency usage conditions from Physical layer. While scheduling radio resources to various service types, real time QoS information and multi-hop nature of CR MANET are considered in LBPFMH algorithm in order to utilize the entire network. Fig. 1, shows the proposed block diagram.

\subsection{CRMANET Network Model}

This research work assumes a distributed multi-hop CRMANET with $N_{s u}$ secondary users (SUs) nodes and $N_{p u}$ primary users (PUs) nodes. Number of licensed channels (LCs) are represented as $C_{P u}$ and unlicensed channels (UCs) are represented as $C_{\mathrm{su}}$. Own infinite buffer is given with all the channels. Both PUs and SUs packets can be hold by LC buffer, where SU packets can be hold by UC buffer. 
The First-Come-First-Served (FCFS) scheduling policy is followed in PUs packets. Pre-emptive priority is given to PU for utilizing LC and SU user's transmission is interrupted.

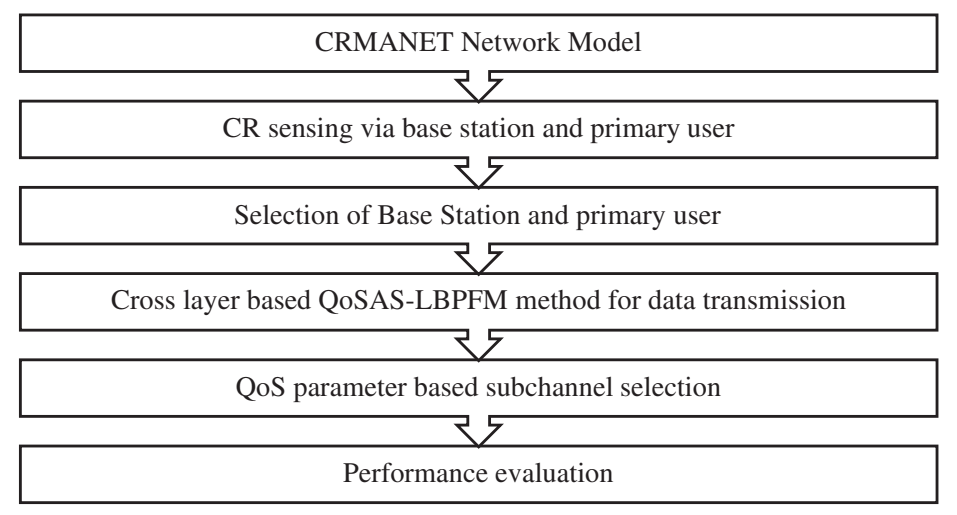

Figure 1: Block diagram of proposed scheduling algorithm

In addition, a Common Control Channel (CCC) is given to all SU. With a time sharing over the main transceiver hardware, dedicated hardware is used for implementing CCC. Control messages like spectrum sensing, routing is received as well as transmitted using this CCC, where reception and transmission are done with a different transceiver. A distributed network is considered with randomly connected links between decentralized nodes and represented as $D(N, L)$, where distribution network is represented as $\mathrm{D}$ and mobile nodes are given as $\mathrm{N}$ and links are represented as L. With network subset, graph set is represented as $N(S)$. Matching is needed if $D$ is a bipartite network with a bipartition of $(I, J)$, as mentioned in graph theory.

\subsection{Base Station Selection}

Connectivity between nodes is used for selecting base station; for giving highly effective communication in CRMANET $(N, L)$, base station is selected from a mobile nodes which is having most links $L$. Control messages are used for counting the links. A control message is broadcasted by every node to its neighbour nodes. Base station is a selected a from a node, which receives a greater number of replies. Request to Send (RTS) message is initially send by channel $C_{x}$ to $N_{n}$, where neighbour nodes are represented as $N_{n}$, where $x=1,2 \ldots n$, and nodes count in that region is represented as $n$.

Then Clear-To-Send (CTS) is send by $N_{n}$ to $C_{x}$ for acknowledgement, if condition $C_{x} \gg C_{(x+1)}$ is true, $C_{x} \rightarrow$ basestation, else $x$ value is increased by 1 and same steps are repeated until satisfying the termination condition. Here, in the routing table, CTS acknowledgements are counted. Own acknowledgment is received by every node and for every node, links count is computed by summing all the received acknowledgements. Using this procedure, comparison is made between links count of every node and base station is selected from a node with high links.

A for loop condition is used for computing a lode with high link count. Using this, for stable condition, this node's mobility is changed as $0 \mathrm{~m} / \mathrm{s}$. Using this stability, link failure is eliminated and achieved the resilience. Base station is used for making a communication between regions 1 and 3 . According to these requirements, saturated vertices are formed by shifting unsaturated vertices. This conversion is also assisted by base station. In a link, information about availability of a spectrum is passed to based station using control messages. 


\subsection{Primary User Selection}

With number of mobile nodes and links, distributed network is given as an input. A bipartite network is expected as an output and data are scheduled to primary as well as secondary users. Initially, various regions are formed by network partitioning [21].

Using this created a network, where every region is given with a primary user, base station and a secondary user. According to node degree, base stations are selected. In a particular region, remaining neighbouring nodes are connected with base station and for every node; coverage range and bandwidth are computed. Ratio between transmission rate and network interface defined the bandwidth.

Bandwidth $=\frac{\text { Transmission rate }}{\sum_{i=1}^{L} N_{i}}$

coverage range $=\sum_{i=1}^{L} \sum_{x=1}^{n}$ area covered by neighbor nodes $\left(N_{x i}\right)$

Area covered by a node defines the coverage range. Selected the primary users according to Eqs. (1) and (2). Using control messages, base station compute this. After the calculation of neighbouring nodes coverage range and bandwidth, node having highest coverage range and bandwidth are checked by base station and corresponding node is assigned as a primary user.

Secondary users are assigned as a reining neighbouring mobile node which is connected with base station. Busy state of primary user indicates that the channel is accessed by primary user to transmit data to the base station. In idle state of primary user, for data transmission, free channels can be accessed by secondary users. Once the primary user and base station is selected then the next step is to perform scheduling schema for data transmission between the nodes.

\subsection{QoSAS-LBPFM Scheduling Scheme}

For QoSAS-LBPFM scheduler, structure of the proposed cross layer is shown in Fig. 2. Before the transmission of packets at every node, packets of every type are given with three buffers. In a similar manner [22], adopted three types of service called Best Effort (BE), Background (BG) and Streaming Media (SM) as typical service in LBPFM algorithm. After the reception of packet, it will be given to buffer process. According to service type, every packet is distributed into one of three service buffers by process buffer. In order to get required QoS related factors for computing priority, end-to-end performance analysis is performed.

For every transmission, repeated the steps in Fig. 2. The limited network resource with some degree of QoS guarantee is utilized by LBPFM via real-time network performance feedback within scheduling algorithm. Ideal CR sensing can be performed using every node of CR MANET and during this time performance cost is not taken into account. Below mentioned QoS conditions needs to be satisfied for enhancing distributed cognitive radio MANET's quality of service.

Here, the QoS factors such as increase in throughput, reduction in delay even with high queuing conditions, avoidance of collision in high level traffic, bandwidth, real-time data users must be given with high priority in scheduling and proper scheduling should be fulfilled. Satisfying every QoS requirement will add a value of one to QoS factor. In ideal conditions, all size conditions will be satisfied and makes quality of service as 6 . LBPFM is performed based on the $i^{\text {th }}$ connection, $N^{\text {th }}$ node's scheduling priority is represented as $S P_{i, N}(t)$ and for various services, QoS factors are used for dynamic update of it. Below mentioned scheduling priority expression is used for SM service. 


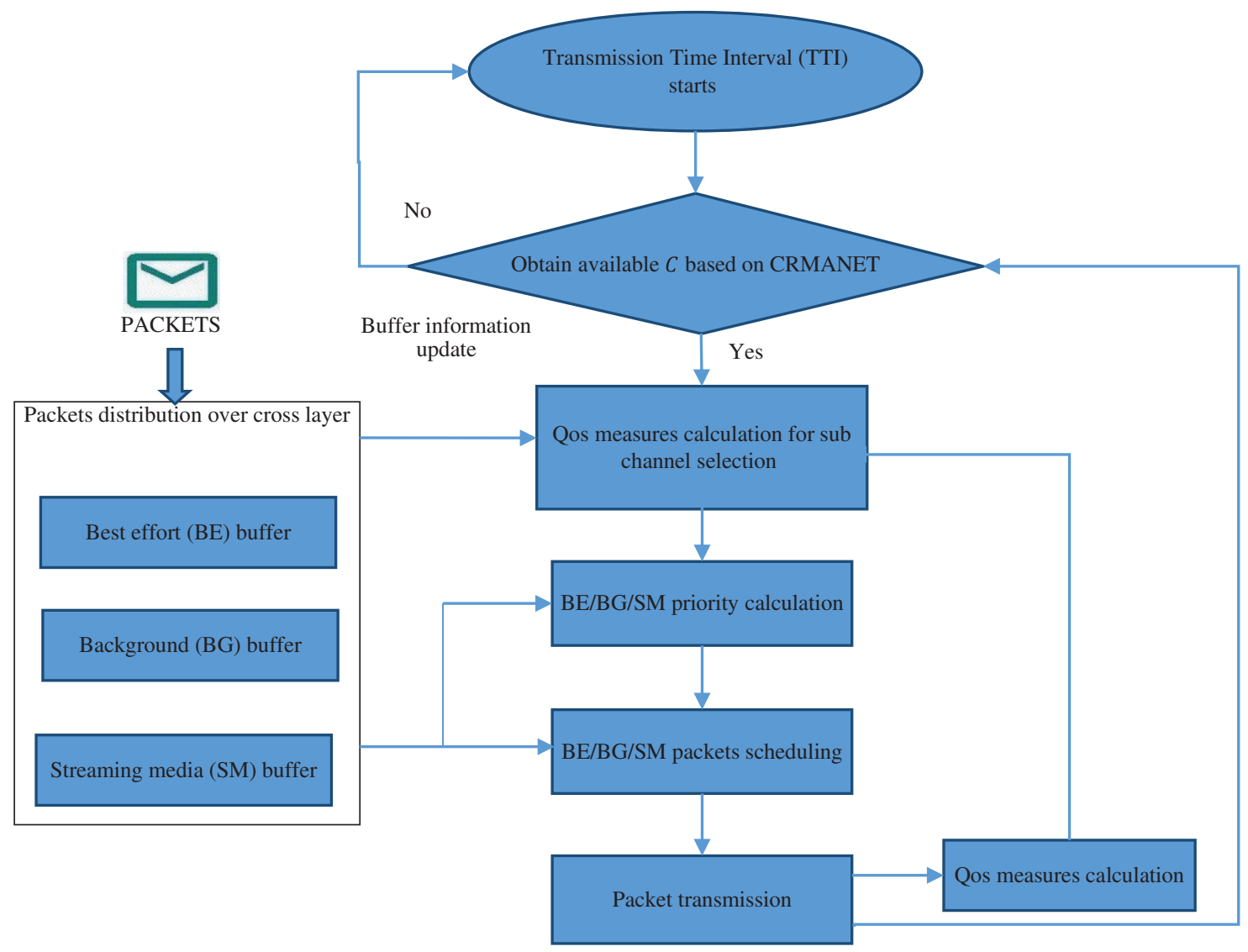

Figure 2: QoSAS-LBPFM scheduler flow diagram

$S P_{i, N}^{S M}(t)=L B P F *$ Throughput $*$ Delay $*$ Bandwidth $* \beta_{S M} *$ traffic

where $L B P F$ represents the Loss Based Proportional Fairness factor, which is used to guarantee scheduling priority for each type of service. Delay factor is represented as Delay and for real-time SM services, an important QoS requirements are represented using this and SM service's initial priority is represented as $\beta_{S M}$. The SM's throughput factor is represented as,

Throughput $=\frac{1}{\frac{\bar{C}_{i}(t+1)}{\min C_{S M}}} \bar{C}_{i}(t+1)=\frac{t}{t+1} \bar{C}_{i}(t)+\frac{1}{t+1} \bar{C}_{i}(t+1)$

where, on a single connection $i$, average throughput till time $t$ is expressed as $\bar{C}_{i}(t)$ and SM service's minimum bit rate requirement is given by $C_{S M}$.

Following expression is used for describing the Delay -delay factor,

Delay $=\left\{\begin{array}{cc}N \log H_{o p}\left(\exp \left(\frac{A W T-D_{i}}{\max D_{i}}\right)+\left(\frac{D_{i}}{\max D_{i}}-1\right)\right) & D_{i}<A W T \leq \max D_{i} \\ N \log H o p_{i} \frac{A V T}{\max D_{i}} & 0 \leq A W T \leq D_{i}\end{array}\right.$ 
$A W T=\frac{\sum_{N=1}^{n} W_{i, N}}{N}$

where, in connection $i$, total waiting time from source node to node $N$ is given as $\sum_{N=1}^{n} W_{i, N}$. Which includes buffers queuing time and transmission time, for this packet, maximum end to end delay tolerance is denoted as max $D_{i}$ and threshold is given by $D_{i}$, which lies between 0 to max $D_{i}$. In real time services like SM, packets will be dropped after reaching max $D_{i}$. Average Waiting Time (AWT), between 0 and threshold $D_{i}$, indicates that the situation is not so urgent.

So, there will be a slow increase in Delay factor, and which results in a slow growth of SM priority. In order to produce better priority, Delay factor will be increased exponentially, if threshold value is exceeded by the average waiting time. In CR MANET, the multi-hop situation is reflected using $\mathrm{N} \log \mathrm{Hop}_{\mathrm{i}}$, in ith connection, Nth node is represented as $\mathrm{N}$.

Along the entire path, packet's location is indicated using this, Priority of SM is increased with the increase in Delay factor as the packet is close to destination node. Total count of connection $i$ is represented as $\mathrm{Hop}_{i}$. High delay factor is produced with path having large number of hops. Traffic prioritization is a type of service and it is different from quality of service. Traffic is generally divided as high, medium and low states. Prioritization is unnecessary with low traffic condition and in order to avoid packet dropping and for reducing delay in high traffic data rate, prioritization needed.

According to real-time and non-real-time data, proper scheduling is allocated by proposed QoS improvement proper scheduling scheme. Only throughput factor is considered a QoS requirement in BG service and BG scheduling priority is expressed as,

$S P_{i, N}^{B G}(t)=$ Throughput $* \beta_{B G}$

Throughput factor in expression (4) and (6) are similar. Only there will be difference in BG packets minimum throughput. BG service's initial priority is given by $\beta_{B G}$. For BE service, there is no prescribed QoS requirements, its Scheduling Priority (SP) is pre-defined priority $\beta_{B E}$.

$S P_{i, N}^{B G}(t)=$ Throughput $* \beta_{B E}$

It is not enough to bypass the cross-layer formulation, from the loss probability attained in a single hop, end-to-end performance is decoupled effectively using this technique. In specific, for s single node, which is fed using a Markovian fluid input traffic and with strict priority scheduling, in buffer size, there will be exponential decay in the ratio between probabilities of strong and the weak class as stated in theory of effective bandwidth.

A packet flow transmitted from any source is assumed to belong to $\mathrm{n}$ possible distinct end-to-end classes as defined in required loss probability value of it. Packet drop probability of class $i$ is $d p_{i}=\alpha_{i} d p_{n}$, for $i=1, \ldots, n$; where, an arbitrary as well as fixed ordered coefficients set is represented as $\alpha=\left(\alpha_{1}, \ldots, \alpha_{n}\right), 0<\alpha_{1}<\alpha_{2}<\ldots<\alpha_{n}=1$ : Class $\mathrm{n}$ having worst performance is made as a reference for other ones, and it is referred as best-effort class. A packet stream is referred as a flow, which carries a similar value on subset of their network, transport or application layer protocol header fields.

With the confirmation of a node's participation along the path between source and destination, available sub-channel(s) up-to-date priority list is created by MAC layer for next hop transmission using the below mentioned procedure. Based on SNR of the available sub-channels, they are stored in a decreasing order. In ordering the sub channels, delay will be considered for the sub-channels having same SNR values. Priority list corresponds to this reordered list. Based on every packet data transmission's QoS requirements, corresponding channels are selected by MAC layer using selection of top channels in the priority list and if further sub-channels are needed, adjacent priority list entries are used. Neighbouring 
table contents of every node is updated after every sensing accordingly. So, re-computation all the values happen and for next packet transmission event, sub-channel list will be refreshed automatically.

As per QoS scheduling detailed in previous section, from top of the priority list $C_{\text {sort }}$, respective channels are selected by MAC layer. Neighbouring table contents of every node is updated after every sensing accordingly. So, re-computation Signal to Noise Ratio (SNR) of every sub-channel happen and for next packet transmission event, priority list $C_{\text {sort }}$ will be refreshed automatically. Tab. 2 states the proposed QoSAS-LBPFM scheme's algorithm. Initially the algorithm starts with network model, then select Base Station (BS) with region R. For that base station then Scheduling Priority (SP) is calculated and allocates channels for data transmission. Then packet transmission phase starts with QoS satisfaction.

Table 2: The Algorithm for the proposed QoSAS-LBPFM scheme

Input: CRMANET $(N, L)$

Output: $\{S P * Q o s\} *\{C R M A N E T(N, L)\} / /$ Scheduling Priority $(S P)$, Quality of Service $(Q o s)$

1. Create $\operatorname{CRMANET}(N, L)$

2. Select Base Satiation $\left(B S_{i}\right)$ based on Request-to-Send (RTS) and Clear-to-Send (CTS) of Channel $C$ with region $\mathrm{R}_{\mathrm{i}}, i=1,2, \ldots n \backslash$ no. of regions and base station Connect

3. Connect $B S_{i}$ to $\mathrm{R}_{\mathrm{i}}(\mathrm{N}), \mathrm{i}=1,2, \ldots \mathrm{n}$

4. Calculate SP by Eq. (7)

5. Send to Queue and allocate $S P$

6. Packet transmission process

7. Return $\mathrm{SP} * \mathrm{CRMANET}(\mathrm{N}, \mathrm{L})$

8. Check $Q o S$

9. If $Q o S \geq 6$ then

10. Return $\{S P * Q o S * C R M A N E T(N, L)\}$

11. Else repeat SP from steps 2 to 5

12. End

\section{Results and Discussion}

A network simulator is used for obtaining the simulation results with a 100 to 500 cognitive nodes which are generated randomly. With different data traffic rates, under Hypertext Transfer Protocol, analyzed the proposed scheme. For data packet transmissions, it used a variable bit rate, User Datagram Protocol and a constant bit rate. Two existing methods called BRACER [23] and QIPS for cognitive radio networks are used for making a comparison with proposed QoSAS-LBPFM scheme. Quality of service parameters like collision probability, efficiency, end to end delay, throughput, delivery rate, overhead ratio is used for computing cognitive radio network performance. Range and details of routing parameters are listed in Tab. 3.

Table 3: Details of routing parameters

\begin{tabular}{ll}
\hline Parameters & Range \\
\hline Routing protocol & AODV \\
Nodes count & 100 to 500 CRMANET \\
Mobility & $20 \mathrm{~m} / \mathrm{s}$ \\
Time of simulation & $200 \mathrm{~s}$ \\
Time of Queuing & $50 \mathrm{~s}$ \\
Type of Queuing & Priority queue \\
\hline
\end{tabular}




\subsection{Throughput Analysis}

Throughput comparison between existing BRACER and QIPS methods and proposed QoSAS-LBPFM scheme are shown in Fig. 3. When compared to other proposed techniques and existing BRACER and QIPS methods, high throughput is produced by proposed QoSAS-LBPFM technique. Here $2 \mathrm{Mbps}$ is allocated as a channel bandwidth and 1.9 Mbps of available spectrum is used by proposed QoSAS-LBPFM technique for 500 no. of nodes. Around $0.6 \mathrm{Mbps}$ throughput is shown by BRACER protocol and $1.7 \mathrm{Mbps}$ is shown by QIPS method for 500 no. of nodes. Increase in nodes count will further increases the throughput performance of a node. This is due to the fact that, in a good scheduling system, sub channels are identified by proposed scheme. This identification makes high throughput with routing table.

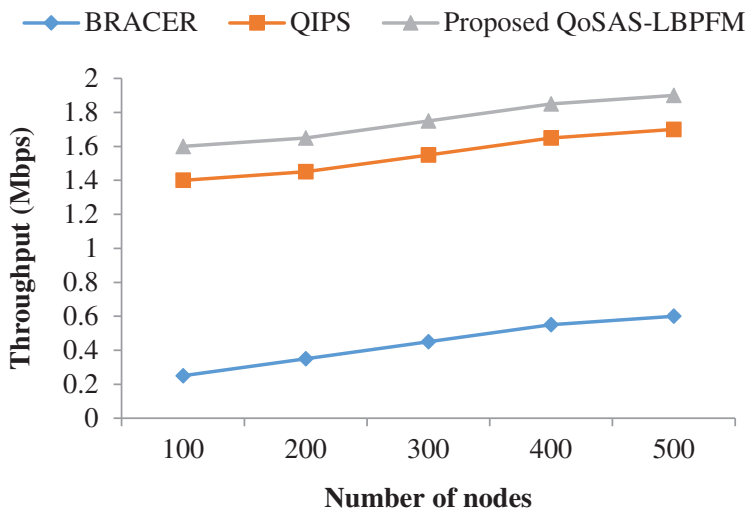

Figure 3: Throughput $v s$. number of nodes

\subsection{Packet Delivery Ratio (PDR)}

Fig. 4 illustrates the delivery ratio comparison. Ratio between number of message delivered and transmitted to destination node defines this delivery ratio. State of transmitted message to destination node portrait in this. When compared BRACER and QIPS techniques, high packet transmission ratio is produced in proposed QoSAS-LBPFM approach. Gradual increase in delivery ration is produced with the increase in number of packets. High delivery ratio of around $87 \%$ is produced by proposed QoSASLBPFM, while $60 \%$ is produced in BRACER broadcast protocol and $83 \%$ is produced in QIPS for 500 no. of nodes. This reduction is due to the high overhead between destination and source.

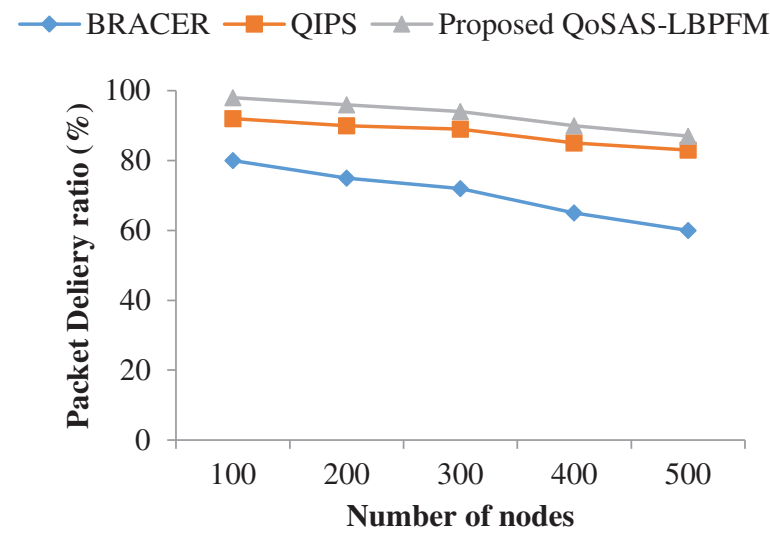

Figure 4: Packet delivery ratio vs. number of nodes 
Collision and prioritization of real time data are reduced in proposed QoSAS-LBPFM scheme for increasing delivery rate, which makes the packet transmission in a fast manner. This is due to the fact that, scheduling priority is identified using LBPFM strategy in the proposed work. Between channel properties, a better coupling is provided by it and flow-level service guarantees are accomplished by circumventing the scheduling algorithms.

\subsection{End-to-End Delay}

With different number of nodes, End-to-end delay comparison of QoSAS-LBPFM, BRACER and QIPS are shown in Fig. 5. Time slot between transmission of data form source to destination including waiting time slot in queuing defines the end-to-end delay. Because of the usage of broadcasting, around 0.6s end-to-end delay is resulted in BRACER broadcast protocol and 0.42s end-to-end delay is resulted in QIPS and there is a chance to lose remaining data due to the QoS-based prioritization for 500 no. of nodes. However, base station in the proposed QoSAS-LBPFM scheme uses service coefficient condition for reducing this delay and it results in $0.33 \mathrm{~s}$ end-to-end delay for 500 no. of nodes. Quick service is given to real-time data applications and in a fast manner, the queue is emptied. For non-real time data, the space of provided by it, and they are transmitted after the transmission of real-time data in the next available slot using LBPFM technique. Feasibility of engineering a prescribed proportional fairness vector based on end-toend loss-based is not affected by interference or random access produced due to incoherent transmission.

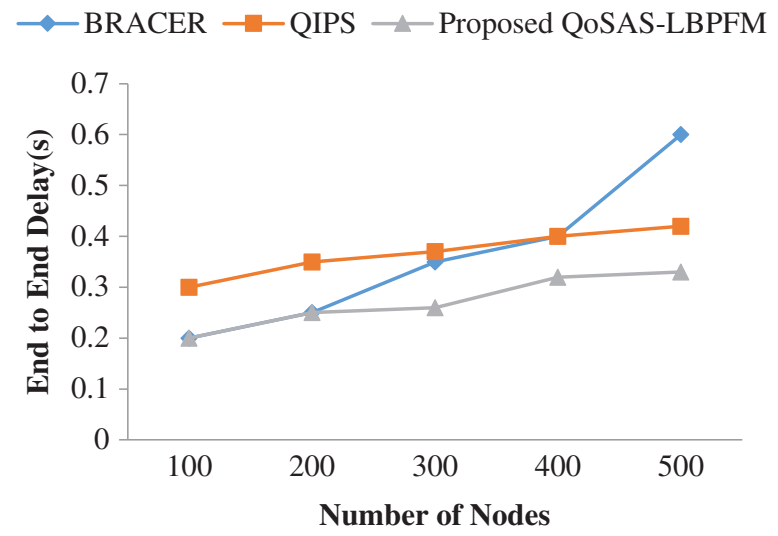

Figure 5: End to end delay vs. number of nodes

\subsection{Network Efficiency}

Relationship between network efficiency of communication and nodes count are illustrated in Fig. 6. Ratio between number of successfully received data packets by the destination to number of successfully transmitted data packets by source after the end simulation defines network efficiency. Because of high delays, efficiency of BRACER broadcast protocol is reduced to $69.00 \%$ and due to high drop rate, efficiency of QIPS is reduced to $69.5 \%$ for 500 no. of nodes. However, drop rate and delay are reduced in the proposed QoSAS-LBPFM model, which increases network efficiency to around $97.00 \%$. Here, service coefficients are used for achieving successful transmission; in addition, base station allocates the secondary users with unused channels immediately for producing better spectrum utilization efficiency, which leads to the increase in network efficiency. If interference and collisions are regarded as a noise by receivers, at the network layer, same packet marking strategy can bale produce proportional differentiation and optimum efficiency of a network can be produced using LBPFM. 


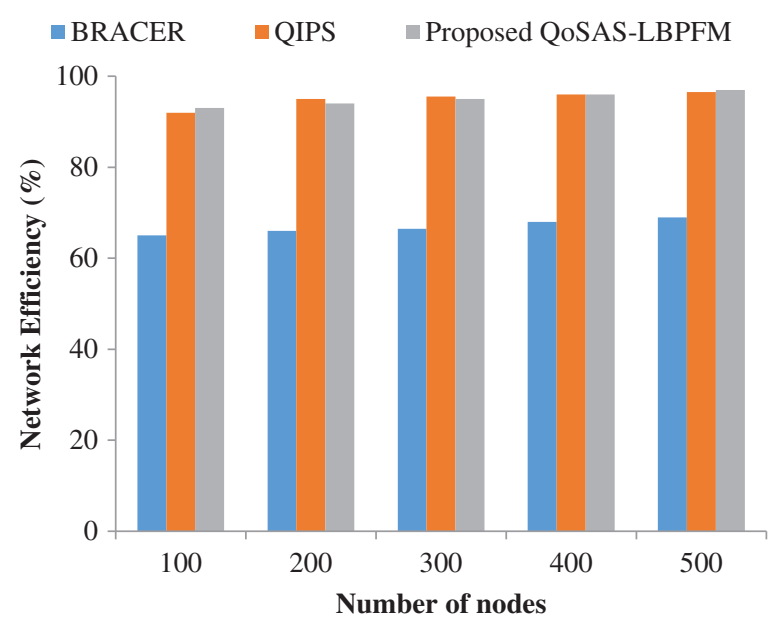

Figure 6: Network efficiency $v s$. number of nodes

\subsection{Collision Probability}

Collision probability comparison is shown in Fig. 7. Ratio between number of packets dropped to number of packets transmitted defines the collision probability. Collision rate will be increased with the increase in drop rate and packet dropping rate and collision probability are in proportion. Message broadcasting of BRACER makes this technique to have high collision probability of around $65 \%$ and QoS-based scheduling in QIPS leads to have high drop rate, which results in a collision probability of around $40 \%$ for 500 no. of nodes. Service coefficient based scheduling in proposed QoSAS-LBPFM model minimizes drop rate as well as collision and around $30 \%$ of collision probability is attained for 500 no. of nodes.

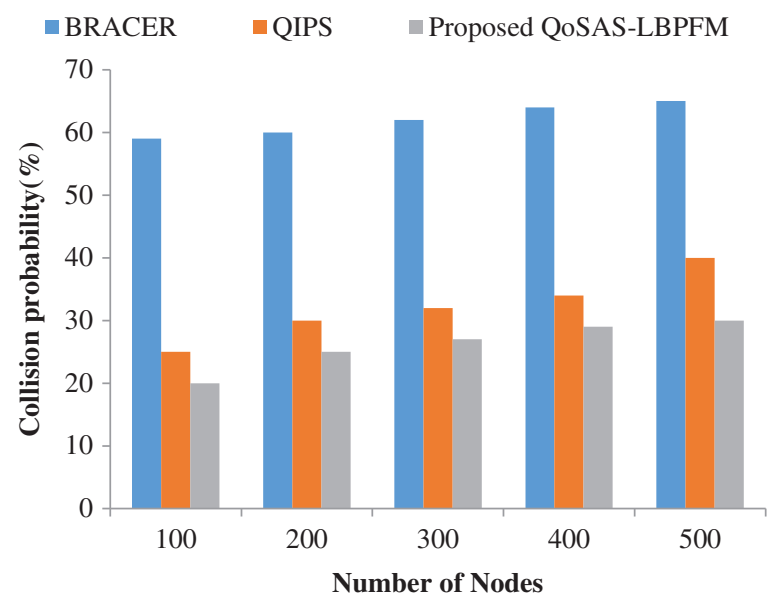

Figure 7: Collision probability vs. number of nodes

\subsection{Routing Overhead Ratio}

Overhead analysis is shown in Fig. 8. Ratio between number of data messages received to number of control messages received defines overhead. Increase in overhead happens with increase in the control messages. In the network, for data transmission between nodes, relatively huge amount of control messages are used in QIPS and BRACER broadcast protocol, which leads to overhead ratio of around 
$42 \%$ and $60 \%$ respectively for 500 no. of nodes. Through a reduced usage of control messages in proposed QoSAS-LBPFM technique, overhead is reduced to $40 \%$ for 500 no. of nodes. Base station is assigned properly for the same as priority detection scheduling and channel assignment of sub channel are done by it. A separate base station is assigned for every region, for converting network's distributed nature into a partially controlled network and reducing overhead.

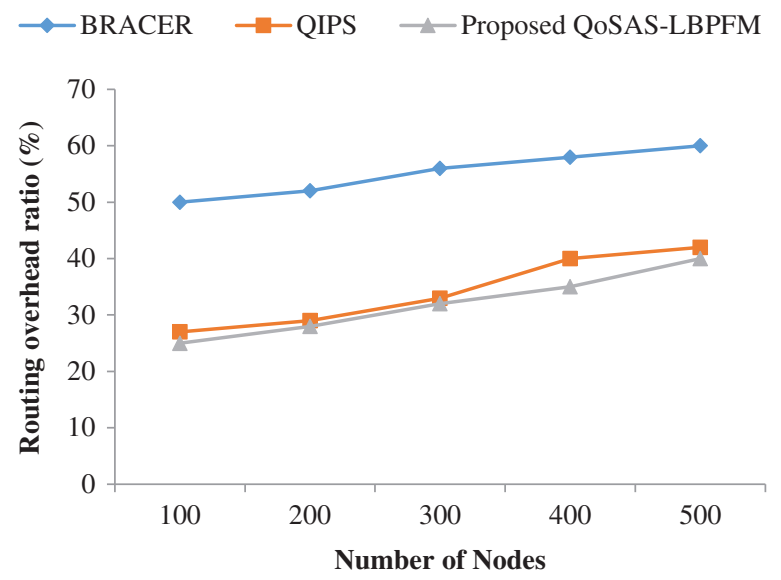

Figure 8: Routing overhead vs. number of nodes

\section{Conclusion and Future Work}

This research work introduced a cross layer QoS scheduling method named LBPFM for CR MANET, that focus to provide requirements of QoS along with stable fairness between varied types of services. Moreover, the multi-channel selection ability in MAC is improvised to utilize the radio sensing information for compensating the noisy surroundings. The present BRACER transmission protocol of CRN have less network performance because of broadcasting data in multi-hop CRN, QoS based prioritization system has reduced network performance because of the utilizing QoS-based prioritization in planning. Introduced QoSAS-LBPFM method provides enhanced network performance by separating the regions of network and providing BS for every region that uses LBPFM related scheduling technique. The BS provides appropriate scheduling for every node in CRN that enhanced the QoS. The outcome of simulation proved that the introduced QoSAS-LBPFM method enhances the efficiency of network and throughput; at the same time lessen the complexity in computation of CR-MANET. From the results it concludes that the proposed system has reduced collision probability $(30 \%)$, end to end delay (0.33 seconds), higher throughput (1.9 mbps), higher delivery rate $(87 \%)$, reduced overhead ratio $(40 \%)$ for CRN with 500 no. of nodes. When many hops are there in the route to destination, the network performance gets reduced regardless of any scheduling technique so implementation of swarm intelligence algorithm is focused for future work.

Acknowledgement: We show gratitude to anonymous referees for their useful ideas.

Funding Statement: The authors received no specific funding for this study.

Conflicts of Interest: The authors declare that they have no conflicts of interest to report regarding the present study. 


\section{References}

[1] Y. Liang, K. Chen, G. Y. Li and P. Mahonen, "Cognitive radio networking and communications: An overview," IEEE Transactions on Vehicular Technology, vol. 60, no. 7, pp. 3386-3407, 2011.

[2] K. Sithamparanathan and A. Giorgetti, "Cognitive radio techniques: Spectrum sensing, interference mitigation, and localization," Artech House, pp. 1-357, 2012. https:/ieeexplore.iee.org/document/9100430.

[3] A. Martian, I. Marcu and I. Marghescu, "Spectrum occupancy in an urban environment: A cognitive radio approach," in Proc. Sixth Advanced Int. Conf. on Telecommunications, Barcelona, Spain, pp. 25-29, 2010.

[4] R. Urgaonkar and M. J. Neely, "Opportunistic scheduling for reliability in cognitive radio networks," IEEE Transactions on Mobile Computing, vol. 8, pp. 1-9, 2010.

[5] D. Xue and E. Ekici, "Delay-guaranteed cross-layer scheduling in multihop wireless networks," IEEE/ACM Transactions on Networking, vol. 21, no. 6, pp. 1696-1707, 2013.

[6] R. Wang, V. K. Lau and Y. Cui, "Decentralized fair scheduling in two-hop relay-assisted cognitive OFDMA systems," IEEE Journal of Selected Topics in Signal Processing, vol. 5, no. 1, pp. 171-181, 2011.

[7] S. Varalakshmi and S. Shanmugavel, "Adaptive retry time based MAC layer spectrum sensing for cognitive radio networks," Asian Journal of Scientific Research, vol. 7, no. 4, pp. 581-590, 2014.

[8] X. Zhang and H. Su, "CREAM-MAC: Cognitive radio-enabled multi-channel MAC protocol over dynamic spectrum access networks," IEEE Journal of Selected Topics in Signal Processing, vol. 5, no. 1, pp. 110-123, 2011.

[9] H. B. Salameh and M. Krunz, "Adaptive power-controlled MAC protocols for improved throughput in hardwareconstrained cognitive radio networks," Ad Hoc Networks, vol. 9, no. 7, pp. 1127-1139, 2011.

[10] J. Park, P. Pawelczak and D. Cabric, "Performance of joint spectrum sensing and MAC algorithms for multichannel opportunistic spectrum access ad hoc networks," IEEE Transactions on Mobile Computing, vol. 10, no. 7, pp. 1011-1027, 2011.

[11] Y. Liang, K. Chen, G. Y. Li and P. Mahonen, "Cognitive radio networking and communications: An overview," IEEE Transactions on Vehicular Technology, vol. 60, no. 7, pp. 3386-3407, 2011.

[12] B. Wang, D. Zhao and J. Cai, "Joint connection admission control and packet scheduling in a cognitive radio network with spectrum underlay," IEEE Transactions on Wireless Communications, vol. 10, no. 11, pp. 38523863, 2011.

[13] T. Luan, F. Gao and X. Zhang, "Joint Resource scheduling for relay-assisted broadband cognitive radio networks," IEEE Transactions on Wireless Communications, vol. 11, no. 9, pp. 3090-3100, 2012.

[14] X. Sun, T. Zhang and D. H. K. Tsang, "Optimal energy-efficient cooperative sensing scheduling for cognitive radio networks with QoS guarantee," in Proc. Int. Wireless Communications and Mobile Computing Conf., Istanbul, pp. 1825-1830, 2011.

[15] G. A. Shah, V. C. Gungor and O. B. Akan, "A cross-layer QoS-aware communication framework in cognitive radio sensor networks for smart grid applications," IEEE Transactions on Industrial Informatics, vol. 9, no. 3, pp. 1477-1485, 2013.

[16] A. Homayounzadeh and M. Mahdavi, "Quality of service provisioning for real-time traffic in cognitive radio networks," IEEE Communications Letters, vol. 19, no. 3, pp. 467-470, 2015.

[17] V. K. Tumuluru, P. Wang and D. Niyato, "A novel spectrum-scheduling scheme for multichannel cognitive radio network and performance analysis," IEEE Transactions on Vehicular Technology, vol. 60, no. 4, pp. 1849-1858, 2011.

[18] R. Yu, W. Zhong, S. Xie, Y. Zhang and Y. Zhang, "QoS differential scheduling in cognitive-radio-based smart grid networks: An adaptive dynamic programming approach," IEEE Transactions on Neural Networks and Learning Systems, vol. 27, no. 2, pp. 435-443, 2016.

[19] A. Ali, L. Feng, A. K. Bashir, S. El-Sappagh, S. H. Ahmed et al., "Quality of service provisioning for heterogeneous services in cognitive radio-enabled internet of things," IEEE Transactions on Network Science and Engineering, vol. 7, no. 1, pp. 328-342, 2020. 
[20] K. Zhang, S. Leng, X. Peng, L. Pan, S. Maharjan et al., "Artificial intelligence inspired transmission scheduling in cognitive vehicular communications and networks," IEEE Internet of Things Journal, vol. 6, no. 2, pp. 19871997, 2019.

[21] P. D. Rathika and S. Sophia, "A distributed scheduling approach for QoS improvement in cognitive radio networks," Computers \& Electrical Engineering, vol. 57, no. 1, pp. 186-198, 2017.

[22] R. Chen, W. Liu, J. Li, C. Li and J. Li, "A fair cross-layer resource allocation scheme with diverse QoS guaranteeing," in Proc. Int. Conf. on Wireless Communications, Networking and Mobile Computing, Beijing, China, pp. 1-5, 2009.

[23] Y. Song and J. Xie, "BRACER: A distributed broadcast protocol in multi-hop cognitive radio ad hoc networks with collision avoidance," IEEE Transactions on Mobile Computing, vol. 14, no. 3, pp. 509-524, 2015. 